\title{
D-ZERO CENTRAL CALORIMETER \\ TECHINCAL APPENDIX TO \\ CRYOGENIC PRESSURE VESSELS
}

(FSM SECTION 5032 TA)

\author{
REVISED
}

\section{D-ZERO ENGINEERING NOTE \#3740.214-EN-267}

GT. Mulholland, R.A. Rucinski

Issued: November 19, 1990

Revised: January 291991 
5032TA for CC Cryostat Pressure Vessel Note

November 7,1990, G. T. Mulholl and

Revised January 22,1991, K. J. Krempetz

\subsection{DOCUMENTATION REQUIREMENTS}

\subsubsection{INTRODUCTION}

DO (D Zero) is a large Liquid Argon (LAr) HEP Calorimeter designed to function in the laboratories P-Pbar collider at the DO section of the Tevatron accelerator. It contains $5,000 \mathrm{gls}$. Of $\mathrm{LAr}$ in the CC cryostat, and $3,000 \mathrm{gls}$. in each of two, a north and south, EC cryostats.

These low pressure vessels are filled with detector modules built of stainless steel, copper and depleted uranium. The LAr functions as the ionization medium, and the spatial and temporal of the collection of the charge of the electrons produced signals the passsage of charged particles. The collection of these charges in $4 \mathrm{pi}$ is related to the energy of the particles, and their measurement is called calorimetry. The contained LAr $(T=90 \mathrm{~K})$ is isolated from the ambient temperatures in specially designed, vacuum and superinsulated, vessels (cryostats) provided with liquid nitrogen, heat of vaporization, cooling.

\subsubsection{FLOW SHEETS}

System flow sheets, 3740-ME-222394, Rev. U, sheets 1 and 2, where delivered to the safety committee on $11 / 6 / 90$.

\subsubsection{PRELIMINARY OPERATING PROCEDURES}

Preliminary procedures will be provided $11 / 15 / 90$. The detail review of the Operating Procedures group is still in progress. The final, preoperation, Operating Procedures will be provided on a schedule consistent with the initiation of cooldown.

\subsubsection{PERSONNEL QUALIFICATION AND TRAINING}

The personnel qualification and training of each lead operator has been in Bubble Chamber operation (see paragraph 4.3 for detail), and each functioned in that capacity for a decade or more. It is anticipated the lead and auxillary operators will commission the system and provide a tested and proven operating procedure after one cooldown, fill, and drain cycle. In the second and subsequent fills these operators will carefully and fully train others in the, then, tried and tested procedures (see 4.2).

\subsubsection{PARTICULAR DOCUMENTATION}

These requirements are answered in the "14.1" written for the cryostat. The pressure vessel and vacuum vessel safety notes by Rucinski and Luther, 3740.214-EN-263, have been completed and are in the review at this time.

\subsubsection{PIPING COMPONENTS LIST}

The list is in DO Piping Components, 3740.510-EN-193, D. Clark, K. Dixon, T. Serges, G. Ball, and J. Michael, delivered to the Safety committee on 11/7/90. 


\subsection{ANALYSIS REQUIREMENTS}

\subsection{FAILURE MODE and EFFECTS ANALYSIS}

The FMEA will be appended to this document.

\subsection{WHAT IF ANALYSIS}

The What-if will be appended to this document.

\subsection{HAZARD ANALYSIS}

The Hazards analysis is in the form of an ODH analysis, 3740.510

$-E N-258, D$. Clark and J. Michael.

\subsection{ENGINEERING CALCULATIONS}

\subsection{RELIEF DEVICE ADEQUACY}

The adequacy of relief devices is addressed in 1.1 .5 above.

\subsection{STRESS LEVELS}

Flexibility and stress calculations for the cryostat piping are documented in DO Engineering Note 3740.210-EN-25.

\subsection{OTHER CALCULATIONS}

The only warm structural component having safety implications is the carriage which supports the cryostat on the center beam of the platform. The carriage is a welded structure designed in accordance with the rules of the AISC Specification for Structural Steel for Buildings (by hand calculations). The design was then analyzed for deflections using finite element methods, which also served to verifiy the stress levels in the stuctural members. Upon delivery, the carriage was load-tested to $125 \%$ of its design loading and deflections were measured at the design load. The design and the finite element analysis are contained in Engineering Note 3740.215-EN-14, and the measured deflections are documented in Appendix A of Engineering Note 3740.214-EN-103. These Notes were submitted for review to the Cryosafety Review Panel as part of the standard documentation procedure and were accepted by the Panel on 3-30-87 and 12-18-87. The Notes were not submitted to the Mechanical Safety Review Panel for their approval.

\subsection{MAINTAINING SAFE OPERATION}

\subsection{DOCUMENTS}

The DO cryogroup has one of the most extensive Engineering Note, Drawing, and Operating Procedures files at the laboratory. It will be maintained and augmented as required to continue this high level of support documentation.

\subsection{PLANS}

The DO Cryosystem is monitored and controlled by a TI565, Programmable Logic Controller. All of the sensor and controls work has been designed to support unmanned steady state (exclusive of cooldown) LAr operation. The sysytem is monitored by 1) a link to the main Do control room, 2) the laboratory FIRUS system, and 3) a cryoexpert autodialing system. After the initial runnning the experience DO personnel will conduct training and quailfication classes for a broader group of operators in RD Cryo. Long term running would be attended, only as required, by this group. The Cryoexperts would continue in their role for the foreseeable future. 


\subsection{TRAINING}

The designers and builders are training themselves for the initial operation. Formal training classes will subsequently be delivered to all potential operators. Only those operators that have successfully completed the formal classes and a rigorous $0 \mathrm{JT}$ period will be added to the qualified operator list. Cryoexperts wi il be trained as necessary to keep a ready supply on hand for each running period.

The Cryoexpert and lead operators are listed below;

\section{NAME}

*****

Dixon, Kelly

Markley, Dan

Rucinski, Russ

Cross, Pete

ALTERNATES

**********

Ramirez, Ernie

Urbin, John

Krempetz, Kurt
EXPERIENCE

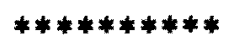

10 years

10 years

2 years

11 years

10 years

10 years

13 years

\subsection{INSPECTIONS}

\subsection{INITIAL}

DO cryo will provide an initial walk through inspection and answer any safety related question the DO Cryosafety panel may have on a schedule of their choosing.

\subsection{SUBSEQUENT}

The DO Cryosafety panel is encouraged to schedule visits to the facility to assure the cryosafety provisions are, in fact, in place and functional. 
R. Rucinski $11 / 19 / 90$

Rev. 1; $1 / 29 / 91$

\section{D-ZERO CENTRAL CALORIMETER}

\section{FMEA ANALYSIS}

This failure mode and effects analysis was done in accordance with the Fermilab safety manual section 5032TA appendix A. Flow diagram drg. no. 3740-ME-222394 rev. U was used. Revision 1 updates the analysis thru rev. $\mathrm{V}$ of the above flow diagram.

\section{Scope:}

All components related to operating the central calorimeter were included. Valves and other components located on the platform or in the cryocorner were included. Components relating specifically to the end calorimeters were excluded. Pipe failures were excluded, they are dealt with in the ODH analysis in D-Zero engineering note 3740.510-EN-258, D. Clark and J. Michael. Instrument air valves and components were excluded. See the "What if" analysis for consequences from loss of instrument air.

\section{Definitions:}

Safe - No mechanical damage or personnel injury.

ODH - Releases argon or nitrogen to atomosphere, possibly threatening personnel. This possibility is taken into acount in the ODH analysis.

Mech. Damage - Possible damage to equipment, most likely due to overpressurization. Personnel injury is considered sufficiently unlikely and as such, constitutes an acceptable risk.

Unsafe More than negligible possibility of personnel injury even if standard ODH procedures are followed.

Note: Failure rates for relief valves taken from Fermilab Standard 5064TA, Table III. 


\begin{tabular}{|c|c|c|c|c|c|c|}
\hline \multicolumn{3}{|c|}{ COMPONENT } & \multirow{2}{*}{$\begin{array}{l}\text { FALLURE OR } \\
\text { ERROA MODE }\end{array}$} & \multirow[t]{2}{*}{ HAZARD OA EFFECT } & \multirow{2}{*}{$\begin{array}{c}\text { MAZARD } \\
\text { CLASS }\end{array}$} & \multirow[t]{2}{*}{ REMARKS OR RECOMENDATIONS } \\
\hline & & & & & & \\
\hline $\mathrm{RO}$ & 136 & $\mathrm{~N}$ & gets clogged & Lose exhaust purge flow & Safe & Will have possibility of Ar freezing in exhaust line. \\
\hline \multirow[t]{4}{*}{ PV } & 201 & $N$ & Fails to open & Lose Ln2 to cooldown condenser & Safe & \\
\hline & & & close unexpect. & Lose Ln2 to cooldown condenser & Safe & \\
\hline & & & Fails to close & Lower pressure in the CC & Safe & Low CC pressure would alarm. Could use PV-513N to limit LN2 flow. \\
\hline & & & open unexpect. & Lower pressure in the $\mathrm{CC}$ & Safe & Low $C C$ pressure would alarm. Could use PV-513N to limit LN2 flow. \\
\hline \multirow[t]{4}{*}{ PV } & 202 & $\mathrm{~N}$ & Fails to open & Lose Ln2 to S.S. operating condenser & Safe & \\
\hline & & & close unexpect. & Lose Ln2 to S.S. operating condenser & Safe & \\
\hline & & & Fails to close & Lower pressure in the $\mathrm{CC}$ & Sale & Low CC pressure would alarm. Could use PV-513N to limit LN2 flow. \\
\hline & & & open unexpect. & Lower pressure in the $\mathrm{CC}$ & Safe & Low CC pressure would alarm. Could use PV-513N to limit LN2 flow. \\
\hline \multirow[t]{2}{*}{ MV } & 203 & A & Open & Normal & Safe & \\
\hline & & & Closed & PT-204A blocked & Safe & CC pressure is indicated by PT-230A also. \\
\hline PT & 204 & A & falls & Lose CC pressure indication & Sale & CC pressure is indicated by PT-230A also. \\
\hline \multirow[t]{2}{*}{$\mathrm{MV}$} & 205 & A & Open & Capped after outlet & $\mathrm{OOH}$ & CC gas sample port. No safety hazard involved even withol $t$ cap. \\
\hline & & & Closed & Normal & Sale & \\
\hline$H x$ & 206 & $\mathrm{~N}$ & Rupture & Cause CC to relieve & Safe & Relief capacity of $C C$ is adequate to handle this case. See EN-263 \\
\hline$H x$ & 207 & $\mathrm{~N}$ & Rupture & Cause CC to relieve & Safe & Relief capacity of $\mathrm{CC}$ is adequate to handle this case. See $\overline{\mathrm{N}}-263$ \\
\hline \multirow[t]{4}{*}{ PV } & 208 & $\mathrm{v}$ & Fails to open & Unable to establish CC vacuum & Safe & Would be discovered before anything was put in the $\mathrm{CC}$. \\
\hline & & & close unexpect. & Unable to establish CC vacuum & Safe & Would be discovered before anything was put in the $\mathrm{CC}$. \\
\hline & & & Fails to close & Will not be able to use VP246V & Safe & Would be discovered before anything was put in the $\mathrm{CC}$. \\
\hline & & & open unexpect. & $\mathrm{CC}$ insulating vacuum gets soft & Safe & Insulating vacuum would be on order $10 \mathrm{E}-4$ torr instead $10 \mathrm{E}-6$ torr \\
\hline \multirow[t]{2}{*}{ PSV } & 209 & $\mathrm{v}$ & Fails to relieve & Pressure in the CC Vacuum Vessel & Sale & Lift plate. Relief on vacuum piping would open. 12 level fallure case] \\
\hline & & & open unexpect. & Lose CC insulating vacuum & Safe & Relief capacity of $\mathrm{CC}$ is adequate to handle this case. See $\mathrm{EN}-263$ \\
\hline PT & 210 & $\mathrm{~N}$ & fails & Lose indication of condensor pressur & Sale & Affects PV-210N, see PV-210N failure mode effect analy: is \\
\hline \multirow[t]{4}{*}{ PV } & 1210 & $\mathrm{~N}$ & Fails to open & Raise $\mathrm{CC}$ condensor temperature & Safe & PSV-211N would relieve. CC pressure would build very sliwly. \\
\hline & & & close unexpect. & Raise $\mathrm{CC}$ condensor temperature & Safe & PSV-211N would relieve. CC pressure would build very skiwly. \\
\hline & & & Fails to close & Condensors can make LAr rain & Safe & CC pressure would decrease. \\
\hline & & & open unexpect. & Condensors can make LAr rain & Sale & CC pressure would decrease. \\
\hline \multirow[t]{2}{*}{ PSV } & 1211 & $\mathrm{~N}$ & Fails to relieve & Overpressure condensing coils & Mech. Damage & Failure rate: Fail to open 1 in 100,000 demands. [2 level failure case] \\
\hline & & & open unexpect. & Vents nitrogen & $\mathrm{OOH}$ & Vents inside building \\
\hline \multirow[t]{2}{*}{ Fol } & 212 & A & Fails to rupture & Will not back up a PSV-213A failure & Safe & RD-212A is not needed for any reliel case. [ 3 level failure case] \\
\hline & & & Rupture & Will vent $\mathrm{CC}$ outside & Safe & \\
\hline
\end{tabular}




\begin{tabular}{|c|c|c|c|c|c|c|}
\hline \multicolumn{3}{|c|}{ COMPONENT } & \multirow{2}{*}{\begin{tabular}{|l} 
FAILURE OR \\
ERPOR MODE
\end{tabular}} & \multirow[t]{2}{*}{ HAZARD OR EFFECT } & \multirow{2}{*}{$\begin{array}{c}\text { HAZARD } \\
\text { CLASS }\end{array}$} & \multirow[t]{2}{*}{ REMARKS OR RECOMENDATTONS } \\
\hline & & & & & & \\
\hline \multirow[t]{2}{*}{ PSV: } & 213 & A & Fails to relieve & CC pressure will raise to $18 \mathrm{psig}$ & Safe & RD-212A will back-up this failure mode. [2 level failure $c$ ase] \\
\hline & & & open unexpect. & Will vent $\mathrm{CC}$ outside & Safe & \\
\hline \multirow[t]{4}{*}{ PV } & 214 & A & Fails to open & Block direct GAr line from Ar dewar & Safe & It would be possible to use the UV piping for GAr leed. \\
\hline & & & close unexpect. & Block direct GAr line from Ar dewar & Safe & It would be possible to use the UV piping for GAr leed. \\
\hline & & & Fails to close & Allows GAr from Ar dewar & Safe & Can stop GAr supply by closing PV-611A or MV-648A. \\
\hline & & & open unexpect. & Allows GAr from Ar dewar & Safe & Can stop GAr supply by closing PV-611A or MV-648A. \\
\hline \multirow[t]{4}{*}{ PV } & 215 & A & Fails to open & Block vac. pumps from $\mathrm{CC}$ pres. vess. & Safe & Can evacuate CC press. vessel thru drain line. \\
\hline & & & close unexpect. & Block vac. purnps from $\mathrm{CC}$ pres. vess. & Safe & Can evacuate CC press. vessel thru drain line. \\
\hline & & & Falls to close & CC press. vess. tied into UV piping & Safe & Can isolate UV piping with MV-454UV. \\
\hline & & & open unexpect. & Vac. pumps tied into CC's cold GAr & Safe & PV-215A is interlocked, can't open with CC positive pressure. \\
\hline \multirow[t]{2}{*}{$\mathrm{mv}$} & 216 & $\mathrm{~N}$ & Open & Normal & Safe & \\
\hline & & & Closed & Blocks PT-210N & Safe & PV-210N will not contral correctly. \\
\hline \multirow[t]{2}{*}{$m v$} & 217 & $\mathrm{~N}$ & Open & Capped after outlet & $\mathrm{COH}$ & Nitrogen sample port trom CC condensers \\
\hline & & & Closed & Normal & Safe & \\
\hline \multirow[t]{4}{*}{$\mathrm{PV}$} & 218 & A & Fails to open & Will not be able to fill $C C$ with LAr & Safe & Will have to fix problem to fill CC with LAr. \\
\hline & & & close unexpect. & Stops LAr fill of CC & Safe & Will have to fix problem to fill CC with LAr. \\
\hline & & & Fails to close & CC LAr is not doubly isolated. & Safe & Would need 2 additional failures for any problem to occur from this \\
\hline & & & open unexpect. & CC LAr is not doubly isolated. & Safe & Would need 2 additional failures for any problem to occur from this \\
\hline \multirow[t]{2}{*}{$\mathrm{cv}$} & 219 & $\mathrm{H}$ & Sticks closed & Blocks He bottles from PV-219A & Safe & Gas bottle GB-219H has helium to operate PV-219A for some time. \\
\hline & & & Sticks open & GB-219H can bleed down & Safe & During a cryostat move could cause PV-219A to open. \\
\hline \multirow[t]{4}{*}{ PV } & 219 & A & Fails to open & Will not be able to fill CC with LAr & Safe & Will have to fix problem to fill CC with LAr. \\
\hline & & & close unexpect. & Stops LAr fill of CC & Safe & Will have to fix problem to fill $C C$ with LAr. \\
\hline & & & Fails to close & CC LAr is not doubly isolated. & Safe & Would need 2 additional failures for any problem to occur from this \\
\hline & & & open unexpect. & CC LAr is not doubly isolated. & Sate & Would need 2 additional failures for any problem to occur from this \\
\hline \multirow[t]{2}{*}{ PSV } & 219 & $\mathrm{H}$ & Fails to relieve & PV-219A would not work & Safe & PRV-219H would have to fail for this failure to occur 12 level lailure \\
\hline & & & Opens unexpect. & Vents helium & $\mathrm{COH}$ & Would increase frequency of changing helium bottles \\
\hline \multirow[t]{2}{*}{ PRV } & 219 & $\mathrm{H}$ & close unexpect. & Lose low pressure He in PV-219A & Safe & PV-219A would still work \\
\hline & & & open unexpect. & Causes PSV-219H to relieve & Sale & PV-219A would still work. Would go thru more He botlles. \\
\hline PS & 219 & $\mathrm{H}$ & fails & Lose indication of PV-219A position & Sale & PS-219H redundantly shows position of PV-219A \\
\hline EV & 219 & H & fails & Lose control of PV-219A & Sale & Can use PV-218A in place of PV-219A \\
\hline 터 & 220 & A & rails & Can't warm up CC contents & Sate & Alternative methods would be possible. \\
\hline \multirow[t]{4}{*}{ PV } & 221 & $\mathrm{v}$ & Fails to open & Would not have VP246V on line & Safe & Insulating vacuum would be on order $10 \mathrm{E}-4$ torr instead $10 \mathrm{E}-6$ torr \\
\hline & & & close unexpect. & Turns off VP246V & Sale & Interlocked, insul. vac. d.p. turns off when PV-221V is closed. \\
\hline & & & Fails to close & Backstreaming into insu. vac. pass. & Safe & PV-225A upstream protects the insul. vac. from backstreaming. \\
\hline & & & open unexpect. & Backstreaming into insu. vac. poss. & Sale & PV-225A upstream protects the insul. vac. from backstreaming. \\
\hline
\end{tabular}




\begin{tabular}{|c|c|c|c|c|c|c|}
\hline \multicolumn{3}{|c|}{ COMPONENT } & \multirow{2}{*}{$\begin{array}{l}\text { FAILURE OR } \\
\text { ERROR MODE }\end{array}$} & \multirow[t]{2}{*}{ HAZARD OR EFFECT } & \multirow{2}{*}{$\begin{array}{c}\text { HAZARD } \\
\text { CLASS }\end{array}$} & \multirow[t]{2}{*}{ REMARKS OR RECOMENDATIONS } \\
\hline & & & & & & \\
\hline DPT & 222 & A & talls & Lose indication of LAr level & Safe & Other devices give LAr level in $c c$. \\
\hline EI & 223 & A & fails & Lose indication of LAr level & Safe & Other devices give LAr level in CC. \\
\hline \multirow[t]{2}{*}{$m v$} & 224 & A & Open & Bypasses DPT-222A & Sale & See DPT-222A fails \\
\hline & & & Closed & Normal & Sale & \\
\hline \multirow[t]{3}{*}{ PV } & 225 & $\mathrm{~V}$ & Fails to open & Isolates VP246V & Safe & Insulating vacuum would be on order $10 \mathrm{E}-4$ torr instead $10 \mathrm{E}-6$ torr \\
\hline & & & close unexpect. & Isolates VP246V & Safe & Insulating vacuum would be on order $10 \mathrm{E}-4$ torr instead $10 \mathrm{E}-6$ torr \\
\hline & & & open unexpect. & Backstreaming into insu. vac. poss. & Safe & No danger involved 2 level failure case] \\
\hline TG & 226 & $\mathrm{~V}$ & fails & Lose indication of D.P. inlet pressure & Safe & \\
\hline$\infty$ & 227 & V & fails & Lose indication of D.P. inlet pressure & Safe & \\
\hline \multirow[t]{4}{*}{ PV } & 228 & A & Fails to open & Isolates the venting of PSV-213A & Safe & If PV-228A doesn't open by operating procedures we will not fill the $\mathrm{CC}$. \\
\hline & & & close unexpect. & Isolates the venting of PSV-213A & Safe & PV-228A will be mechanically locked open. [2 level failure case] \\
\hline & & & Falls to close & Leak thru relief in CC P\&P sequence & Safe & \\
\hline & & & open unexpect. & Leak thru relief in CC P\&P seguence & Safe & \\
\hline El & 229 & $E$ & fails & Lose RD outlet temp. indication & Safe & \\
\hline $\mathrm{PT}$ & 230 & A & fails & Lose CC pressure indication & Safe & PV-214A will not control correctly. PT-204A indicates CC pressure. \\
\hline$\infty$ & 231 & V & fails & Lose CC insul. vac. pressure ind. & Safe & \\
\hline TS & 232 & $E$ & fails & Lose temp. ind. of condenser outlet & Safe & May waste some LN2 without this temperature switch. \\
\hline $\mathrm{PT}$ & 233 & A & lails & Lose VPT pressure indication & Safe & \\
\hline PT & 234 & A & lails & Lose VPT pressure indication & Safe & \\
\hline DPT & 235 & A & lails & Lose LAr temp. gradient information & Sale & \\
\hline $\mathrm{FO}$ & 236 & $N$ & gets clogged & Lose exhaust purge flow & Sate & Will have possibility of Ar treezing in exhaust line. \\
\hline \multirow[t]{2}{*}{ MV } & 237 & A & Open & Bypasses DPS238A & Sale & Lose ind. of LAr flowrate at CC. FM671A indicates llowrate at LAr dewar. \\
\hline & & & Closed & Normal & Safe & \\
\hline DPS & 238 & A & lails & Lose CC LAr flow rate information & Safe & FM671A indicates flowrate at LAr dewar \\
\hline$F M$ & 239 & A & lails & Lose CC LAr flow rate information & Sale & FM671A indicates flowrate at LAr dewar \\
\hline PT & 240 & A & lails & Lose VPT pressure indication & Safe & \\
\hline \multirow[t]{2}{*}{$\mathrm{mv}$} & 241 & A & Open & A cap looks at CC GAr & $\mathrm{OOH}$ & \\
\hline & & & Closed & Normal & Safe & \\
\hline $\mathrm{mv}$ & & & Closed & Normal & Sale & \\
\hline \multirow[t]{2}{*}{ mv } & 244 & A & Open & A cap looks at $C C \mathrm{GAr}$ & $\mathrm{OOH}$ & \\
\hline & & & Closed & Normal & Sale & \\
\hline
\end{tabular}




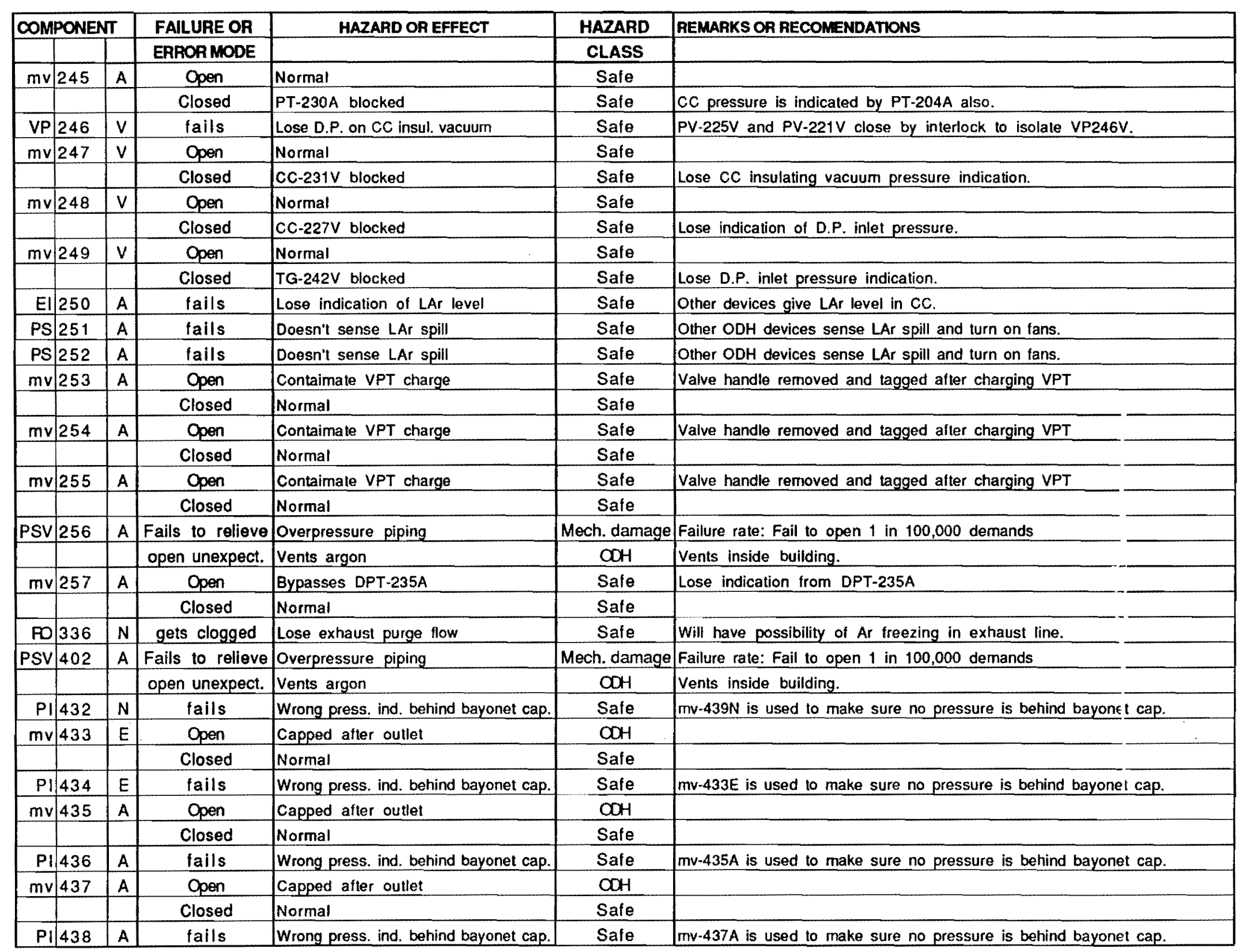




\begin{tabular}{|c|c|c|c|c|c|c|}
\hline \multicolumn{3}{|c|}{ COMPONENT } & \multirow{2}{*}{$\begin{array}{l}\text { FAILURE OR } \\
\text { ERROR MODE }\end{array}$} & \multirow[t]{2}{*}{ HAZARD OR EFFECT } & \multirow{2}{*}{$\begin{array}{c}\text { HAZARD } \\
\text { CLASS }\end{array}$} & \multirow[t]{2}{*}{ REMARKS OR RECOMENDATIONS } \\
\hline & & & & & & \\
\hline \multirow[t]{2}{*}{ mv. } & 439 & $\mathrm{~N}$ & Open & Capped after outlet & $\mathrm{OCH}$ & \\
\hline & & & Closed & Normal & Safe & \\
\hline \multirow[t]{2}{*}{$\mathrm{mv}$} & 453 & $\mathrm{v}$ & Open & Normal & Sale & \\
\hline & & & Closed & Isolates vac. pumps from cryostats & Safe & Cryostat insul. vac. could get soft before this is discoveret? \\
\hline \multirow[t]{2}{*}{ MV } & 454 & UV & Open & Normal & Sare & \\
\hline & & & Closed & Isolates vac. pumps from cryostats & Safe & Would be discovered during P\&P of cryostats. \\
\hline PS & 471 & $\mathrm{~N}$ & fails & Lose pressure indication & Safe & Lose confirmation of warm GN2 purge of exhaust piping. \\
\hline \multirow[t]{2}{*}{$M V$} & 474 & A & Open & Normal & Sale & \\
\hline & & & Closed & Stops Gar flow to cryostats & Sale & Blocks direct GAr feed from LAr dewar. \\
\hline \multirow[t]{2}{*}{ PSV } & 475 & A & Fails to relieve & Overpressure piping & Mech, damage & Failure rate: Fail to open 1 in 100,000 demands \\
\hline & & & open unexpect. & Vents argon & $\mathrm{COH}$ & Vents inside building. \\
\hline \multirow[t]{2}{*}{ MV } & 476 & A & Open & Normal & Safe & \\
\hline & & & Closed & Stops LAr fill to cryostats & Sale & \\
\hline \multirow[t]{2}{*}{ MV } & 477 & $\mathrm{~N}$ & Open & Normal & Sale & Permits LN2 to condensers \\
\hline & & & Closed & Stops LN2 to condensers & Safe & Loss of liquid nitrogen, see "What if" analysis \\
\hline RM & 478 & $\mathrm{~N}$ & falls & Lose LN2 cooldown flowrate info. & Safe & \\
\hline PM & 479 & $\mathrm{~N}$ & falls & Lose LN2 s.s. llowrate into. & Safe & \\
\hline EV & 480 & $\mathrm{~N}$ & fails & Can't switch between flowmeters & Safe & Would lose FM-478N or FM-479N. \\
\hline \multirow[t]{2}{*}{$\mathrm{mv}$} & 481 & $\mathrm{~N}$ & Open & Bypasses DPT-482N & Safe & Lose indication from DPT-482N. \\
\hline & & & Closed & Normal & Safe & \\
\hline DPT & 482 & $N$ & fails & Lose LN2 Howrate information & Sate & Can't tell LN2 flowrate to cryostat condensers. \\
\hline \multirow[t]{2}{*}{$m v$} & 483 & A & Open & Capped after outlet & $\mathrm{OOH}$ & \\
\hline & & & Closed & Normal & Safe & \\
\hline & 484 & $\mathrm{~N}$ & gets clogged & Stops LN2 to condensers & Safe & Loss of liquid nitrogen, see "What if" analysis \\
\hline \multirow[t]{2}{*}{$\mathrm{mv}$} & 485 & $\mathrm{~N}$ & Open & Bypasses DPT-486N & Sale & Lose indication from DPT-486N. \\
\hline & & & Closed & Normal & Safe & \\
\hline DPT & 486 & $\mathrm{~N}$ & fails & Lose press. drop info. & Sale & Won't be able to tell if fither F-484N is getting dirty. \\
\hline EV & 487 & $\mathrm{~N}$ & fails & Can't switch between flowmeters & Safe & Would lose FM- $478 \mathrm{~N}$ or FM- $479 \mathrm{~N}$. \\
\hline \multirow[t]{2}{*}{ MV } & 491 & $\mathrm{~N}$ & Open & Normal & Sate & Permits LN2 to condensors \\
\hline & & & Closed & Stops LN2 to condensers & Safe & Loss of liquid nitrogen, see "What if" analysis \\
\hline \multirow[t]{2}{*}{ PSV } & 492 & $\mathrm{~N}$ & Fails to relieve & Overpressure piping & Mech. damage & Failure rate: Fail to open 1 in 100,000 demands \\
\hline & & & open unexpect. & Vents nitrogen & $\mathrm{OOH}$ & Vents inside building. \\
\hline \multirow[t]{2}{*}{$\mathrm{mv}$} & 493 & $N$ & Open & Capped after outlet & $\mathrm{OOH}$ & \\
\hline & & & Closed & Normal & Safe & \\
\hline
\end{tabular}




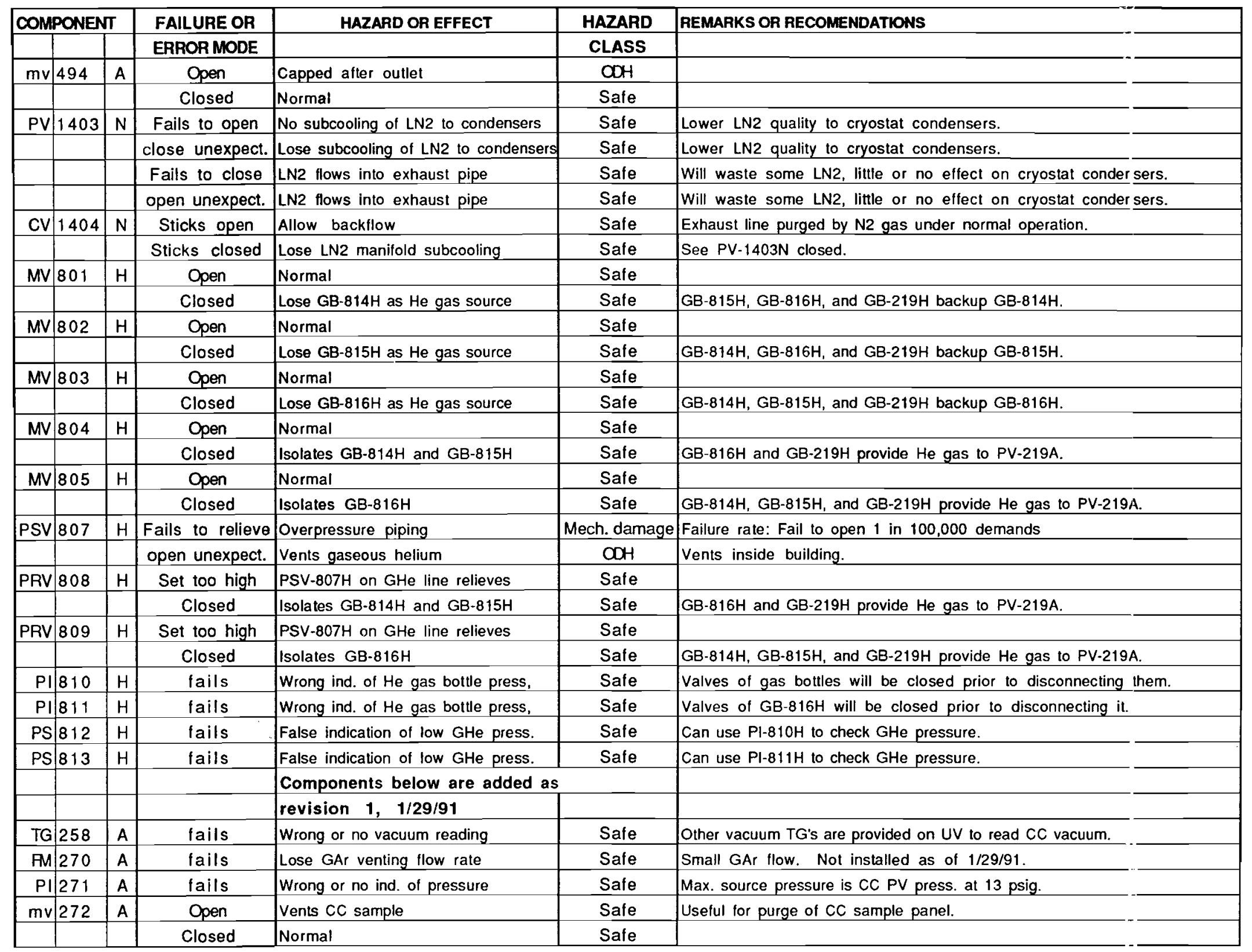




\begin{tabular}{|c|c|c|c|c|c|c|}
\hline \multicolumn{3}{|c|}{ COMPONENT } & \multirow{2}{*}{\begin{tabular}{|l|} 
FAILURE OR \\
ERROR MODE \\
\end{tabular}} & \multirow[t]{2}{*}{ HAZARD OR EFFECT } & \multirow{2}{*}{$\begin{array}{c}\text { HAZARD } \\
\text { CLASS } \\
\end{array}$} & \multirow[t]{2}{*}{ REMARKS OR RECOMENDATIONS } \\
\hline & & & & & & \\
\hline \multirow[t]{2}{*}{$\mathrm{mv}$} & 273 & A & Open & Lose a GAr sample & Safe & Port for taking a remote GAr sample. \\
\hline & & & Closed & Normal & Safe & \\
\hline \multirow[t]{2}{*}{$m v:$} & 274 & A & Open & Connects vacuum to $\mathrm{CC}$ sample panel & Safe & Pumping port for $\mathrm{CC}$ sample panel. \\
\hline & & & Closed & Normal & Safe & \\
\hline \multirow[t]{2}{*}{$\mathrm{mv}$} & 275 & A & Open & Allows sample to CC sample panel & Safe & mv241A is in series to CC press. vessel. \\
\hline & & & Closed & Normal & Safe & \\
\hline \multirow[t]{2}{*}{$\mathrm{mv}$} & 276 & A & Open & Allows sample to CC sample panel & Safe & mv243A is in series to CC press. vessel. \\
\hline & & & Closed & Normal & Safe & \\
\hline TG: & 277 & A & fails & Wrong or no vacuum reading & Safe & The $C C$ sample panel will be purged out and is isolated from the $C C$ \\
\hline PS & 826 & $\mathrm{H}$ & fails & Lose alarm signal to control system & Sale & Will lose one of the two cold valve helium supply alarms. \\
\hline \multirow[t]{2}{*}{$\mathrm{mv}$} & 825 & $\mathrm{H}$ & Open & Normal & Safe & \\
\hline & & & Closed & Isolates PS813H & Sale & See PS813H fails \\
\hline
\end{tabular}


R. Rucinski $11 / 15 / 90$

\section{D-ZERO CENTRAL CALORIMETER "WHAT IF" ANALYSIS}

This "What If" analysis was done in accordance with the Fermilab safety manual section 5032TA appendix B. 


\begin{tabular}{|c|c|c|}
\hline "WHAT - IF" & CONSEQUENCE / HAZARD & CONCLUSION / RECOMMENDATIONS \\
\hline Leaks occur? & Oxygen Deficiency Hazard may occur due to & Leaks of reasonable size have been anticipated by the $\mathrm{ODH}$ analysis and \\
\hline & cryogens leaking into the building. & appropriate provisions made so they present no personnel or \\
\hline & & equipment danger (i.e. the $\mathrm{ODH}$ class is 0 ). \\
\hline \multirow{5}{*}{ The vacuum of the $C C$ is spoiled? } & Loss of vacuum. & The loss of vacuum of the cryostat is a relief case looked at in Do engineering \\
\hline & & note 6 . A heat leak of approx. $3.5 \mathrm{KW}$ will occur causing an additional loading \\
\hline & & of the nitrogen condensors. The required relief capacity for LOV is $139 \mathrm{sclm}$. \\
\hline & & The relief valve and piping on the $C C$ has a capacity of $942 \mathrm{sctm}$ \\
\hline & & plus an additional rupture disc. \\
\hline \multirow{2}{*}{$\begin{array}{l}\text { There is a loss of vacuum on a } \\
\text { transfer line? }\end{array}$} & Increased heat load, lower quality of transferred & There is no sately hazard if this occurred. The problem would be discovered by \\
\hline & liquid, would frost up the effected piping. & either a visual inspection of frost and/or questionable operational behavior. \\
\hline \multirow{4}{*}{$\begin{array}{l}\text { There is a fire under or around } \\
\text { the CC? }\end{array}$} & The CC could relieve and possible loss of & Fire exposure of the CC is looked at in Do Engineering Note 6 . \\
\hline & signal or valve operators could occur. & The required relief capacity for fire is $264 \mathrm{scfm}$. The relief valve and \\
\hline & & piping on the $\mathrm{CC}$ has a capacity of $942 \mathrm{scfm}$ plus an additional rupture disc. \\
\hline & & See also the "What if" there is a loss of signals or valve operators occurs? \\
\hline \multirow{2}{*}{ There is a fire in the cryocorner? } & Could lose electrical signals to and from the $\mathrm{CC}$. & See the "What if" there is a loss of signals or valve operators occurs? \\
\hline & Some of the o-rings on bayonets could fail. & and "What if" a leak occurs? \\
\hline \multirow{2}{*}{$\begin{array}{l}\text { There is a fire in the Ar dewar } \\
\text { room? }\end{array}$} & Could lose electrical signals to and from the $\mathrm{CC}$. & See the "What if" there is a loss of signals or valve operators occurs? \\
\hline & Some of the o-rings on bayonets could tail. & and "What if" a leak' occurs? \\
\hline \multirow{3}{*}{$\begin{array}{l}\text { There is a loss of signals or valve } \\
\text { operators? }\end{array}$} & We would lose the ability to control valves. & All control valves fail in the closed positions. All reliefs and rupture discs \\
\hline & & remain operational. After some time trapped volume reliefs could relieve. \\
\hline & & The CC could relieve also after a long period of time. \\
\hline \multirow{6}{*}{$\begin{array}{l}\text { A cap on an EC rotating bayonet is } \\
\text { missing or is not clamped on? }\end{array}$} & Would not be able to successfully pass pump and & It is written into the CC procedures pump and purge section 7.3 .0 that \\
\hline & purge part of the CC procedures. & the caps are to be installed. If a cap is missing we would not successfully \\
\hline & & be able to pull a vacuum on that line. If a cap was in place but not clamped, \\
\hline & & then it would become unseated after the first backfill to positive pressure \\
\hline & & and the subsequent pumpdown would fail. In either case, the problem \\
\hline & & would be discovered belore cryogens entered the line. \\
\hline
\end{tabular}




\begin{tabular}{|c|c|c|}
\hline "WHAT - IF" & CONSEQUENCE / HAZARD & CONCLUSION/RECOMMENDATIONS \\
\hline \multirow{7}{*}{ There is an earthquake? } & Damage to the piping system could occur. & The ANSI 831.1-1986 pioing code under par. 101.5.3 states that \\
\hline & & "The effect of earthquakes, where applicable, shall be considered in the \\
\hline & & design of piping, piping supporis..." The effect of earthquakes are not \\
\hline & & applicable to the region of lilinois where Fermilab is located. The \\
\hline & & Unilorm Building Code shows that Fermilab is in a Class 0 \\
\hline & & seismic zone which means no design provisions for earthquakes are \\
\hline & & required. \\
\hline \multirow{5}{*}{$\begin{array}{l}\text { There is a loss of electrical } \\
\text { power? }\end{array}$} & Backuo oower will be reouired to maintain & Sustained loss of electrical power will result in critical eouioment \\
\hline & operation of the system. & running on the Emergency Power Generator. The instrument air. \\
\hline & & vacuum pumps and controls are (will) all be EPG powered. Should \\
\hline & & the EPG lail belore the return of commercial power the equipment \\
\hline & & is lost, see below. \\
\hline & & 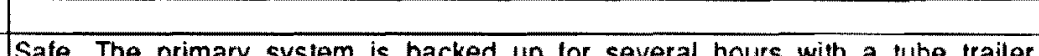 \\
\hline There is a loss of instrument & Valves will close. & Sale. The primary system is backed up lor several hours with a tube trailer \\
\hline \multirow[t]{2}{*}{ air? } & & All valves are failsate, 1.e. they close on the loss of instrument \\
\hline & & air. Reference the failure mode and effects analysis. \\
\hline There is a loss of cooling & The main cooling water suppiy might be & The vacuum and Instrument air equipment have a primary, emergency \\
\hline \multirow{5}{*}{ water? } & in leopardy if a system was not designed & powered, immediate start, closed loop, fan blown radiator, redundant \\
\hline & properly. & pump. glycol stream to put the heat load on the building system and \\
\hline & & provide lower summertime coolant temperatures. Loss of the secondary \\
\hline & & system, or commercial power if the emergency generator functions, \\
\hline & & does not effect the cooling provided to the rotary equipment. \\
\hline & & \\
\hline There is a loss of Liquid & Cooling will be lost and will cause the & The loss of liquid nitrogen denies the detector its necessary coooling \\
\hline \multirow{4}{*}{ nitrogen? } & Vessels to warm, boil off, and vent. & and it will pressurize and vent. The rate of loss is calculated to be \\
\hline & & only $0.45 \mathrm{gpm}$ of liquid argon on average, which is very slow. \\
\hline & & The loss of liquid nitrogen does not provide a personnel or equipment \\
\hline & & danger. \\
\hline & & \\
\hline $\begin{array}{l}\text { Some kind of contamination } \\
\text { occurs? }\end{array}$ & The contamination could restrict flows. & $\begin{array}{l}\text { Continued or serious one-time contamination of the coolant stream } \\
\text { with frozen solids will result in a loss of liquid nitrogen, see above. }\end{array}$ \\
\hline
\end{tabular}




\begin{tabular}{|c|c|c|}
\hline "WHAT - IF" & CONSEQUENCE / HAZARD & CONCLUSION / RECOMMENDATIONS \\
\hline There is some kind of & A piece of equipment will stop working. & $100 \%$ redundancy in mechanical forepumps provide for vacuum \\
\hline \multirow[t]{5}{*}{ equipment failure? } & & equipment lailure in the operating mode. The insulating vacuum can \\
\hline & & function with either the cryostat dilfusion pump or the blower. \\
\hline & & Instrument air is backed up by 8 or more hours of high pressure \\
\hline & & gaseous nitrogen. All valves close on air failure. Expected equipment \\
\hline & & modes do not provide a personnel or equipment danger. \\
\hline An operator makes a & Upset of the system may occur. & Any one operator procedural or console error can cause any one \\
\hline \multirow[t]{4}{*}{ procedural error? } & & component to act improperly which is comparable to a component \\
\hline & & failure. Failure of equipment is covered above and lailure of valves \\
\hline & & was covered in the FMEA. A single operator error cannot cause a satety \\
\hline & & problem. \\
\hline
\end{tabular}

